\title{
On the Performance of Mobile WiMAX System: Measurement and Propagation Studies*
}

\author{
Furaih Alshaalan $^{1}$, Saleh Alshebeili ${ }^{2}$, Abdulkareem Adinoyi ${ }^{3}$ \\ ${ }^{1}$ Department of Electrical Engineering, King Saud University (KSU), Riyadh, Saudi Arabia \\ ${ }^{2}$ Prince Sultan Advanced Technologies Research Institute (PSATRI)/STC Chair, KSU, Riyadh, Saudi Arabia \\ ${ }^{3}$ Swedtel Arabia, Riyadh, Saudi Arabia \\ E-mail: falshalan@yahoo.com,dsaleh@ksu.edu.sa, aadinoyi@gmail.com \\ Received August 6, 2010; revised September 13, 2010; accepted October 24, 2010
}

\begin{abstract}
In this paper, we present drive test results for mobile WiMAX system for desert and cosmopolitan terrains where there are few studies reported in the literature. The extensive measurement is performed in the framework of the physical performance of the WiMAX technology which is often considered as a 4G system. Path loss model is fitted for the collected data. The work is unique in the sense that most empirical channel models are produced in regions where the environments (weather, buildings, vegetation, among others) are quite different from the desert terrains that are considered in this study. We also show that shadowing is truly lognormal in $\mathrm{dB}$ and the standard deviation values are calculated for the desert terrain from the measurement data. The measurements are collected using WiMAX BS station, with greenpacket dongle, and NEMO versatile outdoor drive test equipment to evaluate and characterize the performance of the system. The received signal strength indicators measured, are analyzed to complement network design and network optimization for regions where the popular models may not be accurate.
\end{abstract}

Keywords: OFDMA, WiMAX, RSSI, Path Loss Models

\section{Introduction}

Worldwide Interoperability for Microwave-Access (WiMAX) has emerged as wireless access technology that is capable of providing fixed and mobile broadband connectivity. More accurately, WiMAX is a certification mark for IEEE 802.16-based broadband wireless solutions that have passed a set of conformity and interoperability tests defined by WiMAX forum [1]. Their unified approach to testing manufacturers' equipment has ensured that various vendors' products interwork together.

Fixed WiMAX is targeted for fixed and nomadic broadband services while mobile WiMAX are designed to provide high mobility services. While the performance and propagation models of the fixed WiMAX system have been widely studied [2-5], the mobile WiMAX is yet to witness widespread attention. To the best of our knowledge, there are no reports in the literature for mo-

*This work was supported by a grant (No. 09-ELE302) from The Unit of Science and Technology, King Saud University. bile WiMAX performance presented for the desert terrains that are the subject of this paper. A closely related study is reported in [6] although for GSM network, where it is argued that the Okumura-Hata propagation model is not fully suitable for the desert, dry Oman terrains since there is no much rain in Oman. On the other hand, most propagation models are developed in regions with significant amount of rainfall and flourishing vegetation. The fact of this statement is much applicable to most cities in the Kingdom of Saudi Arabia (KSA). The authors in [6] suggest that further improvement of Okumura-Hata model in the open area is necessary. However, their study is limited to GSM900 operating in the 900 $\mathrm{MHz}$ frequency spectrum.

Operators do drive-tests on a continuous basis, collecting signal levels, network quality and performance which are then used to refine empirical propagation models for system-planning and/or existing network optimization [7-9]. Reference [3] provides measurement results for some Italian University campuses in the 3.5 $\mathrm{GHz}$ frequency band where they attempted to extract and refine previous path-loss models for their experimental 
data. Note that the Walfish and Bertoni model is an extension of the COST-231 (and COST-231 Hata model is an extension of the Hata-Okumura model). The accuracy of this model varies depending on the environments.

If the desert terrain is examined carefully, it will be discovered that it is significantly different from the vegetation rich and densely populated region of Tokyo city where Okumura's path-loss model was developed. A good engineering design suggests that these models should be modified for other cities (or at least perform parameter tuning) to capture the possible differences in the propagation behaviors. The importance of a properly chosen path loss model for evaluating the performance of a system is stressed in [10]. We observed that the nature of buildings and structures is remarkably different as well in the sense that almost all buildings in the KSA are of brick type in contrast to plank or wood of most advanced countries. Thus our study will be useful as it represents an extension of mobile WiMAX study to desert terrains.

In this study we have performed a comprehensive measurement study of the desert terrain. Propagation models are fitted for the measured data. Furthermore, in contrast to [6], in our study, we investigate the WiMAX technology (that is competing with 3GPP's long-term evolution (LTE) as beyond $3 \mathrm{G}$ technology [11]) in the $2.5 \mathrm{GHz}$ band. Our measurement efforts can be adopted for network planning and optimization. These efforts have been motivated by the work in [12], among other references. The authors (in reference [12]) have stressed the importance of careful evaluation of technology to aid network design and optimization. However, their study was limited to fixed WiMAX system in contrast to our mobile WiMAX. Among the benefits of our effort for accurate propagation model for desert environment is to assist network operators not to over- or under-engineer their networks, which is not a cost-effective way of managing link budget. For the design of power-efficient networks it is essential that every fraction of decibel (energy) is utilized effectively through adoption of the most suitable model in link budget. This power consciousness is a valuable effort towards reducing power consumption which has both positive environmental and cost impacts.

The remaining discussion in this paper is organized as follows. The network, system set-up and measurement tools are described in Section 2. This is followed by test result presentation and analyses in Section 3. Finally, conclusion is drawn in Section 4.

\section{System Set-Up and Measurement Tool Descriptions}

We used a NEMO test tool-equipped laptop, housed in a vehicle containing a global positioning system (GPS) to synchronize its location with incoming data provided by the WiMAX terminal (Figure 1). The terminal is a greenpacket (WiMAX) receiver. We have used the versatile NEMO outdoor air interface measurement tool that has been widely accepted in the industrial. The outdoor tool, GPS receiver, and WiMAX scanner are connected to the laptop through a USB serial converter. The GPS in conjunction with the NEMO outdoor system is used to measure the distance of the WiMAX receiver from the base station.

The system uses an air interface based on orthogonal frequency division multiplexing (OFDM), which is very robust against multi-path propagation and frequency selective fading. The WiMAX system uses the Frequency Division Duplexing (FDD) mode of transmission. Collection of measurement data was performed using $\mathrm{Wi}$ MAX BS and communications towers with antennas at a height of 17 meters. The roof of the car (i.e., the omni directional antenna) is two meters in height. The measurement data are collected by driving through the city around the WiMAX BS as shown in the representative signal map shown in Figure 2. Each point represents

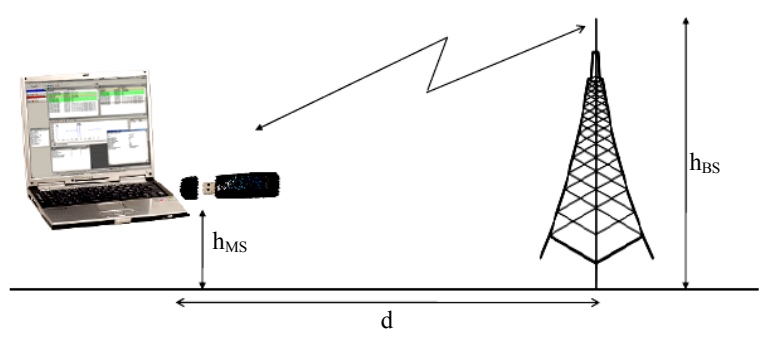

Figure 1. The set-up of the drive test.

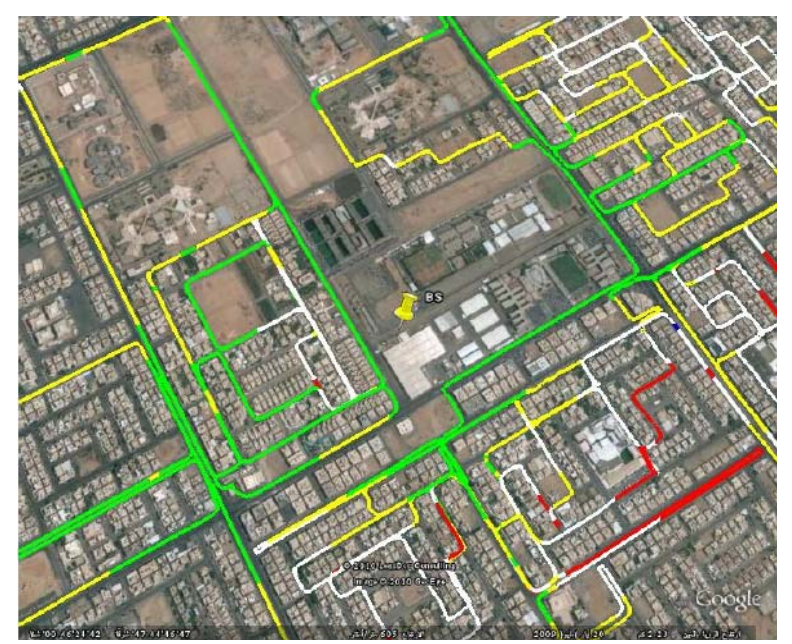

Figure 2. A sample signal map for the collected data for one site. The color legend are Green: $R S S I>-70 \mathrm{dBm}$, Yellow: $-80 \leq$ RSSI $<-70 \mathrm{dBm}$, White: $-89 \leq$ RSSI $<-80 \mathrm{dBm}$, Red: RSSI $<-90 \mathrm{dBm}$. 
sample collected at the position shown. Table 1 shows the summarized specifications for the WiMAX system.

\section{Drive Test Results and Analyses}

The measurement campaign earmarked a number of WiMAX sites. The sites are selected to provide a good mix of various structures that characterize the region's settlements that are the subject of this study. For instance, Riyadh is a fast growing and developing metropolitan and in the suburb, there are pockets of new settlements that are connected by open and mostly desert areas. Thus, this terrain provides an unique environment for new measurement study and more importantly, as far as we know, there has been no study of this kind reported in the open literature.

A sample signal map is shown in Figure 2 where signal level distribution is depicted. As expected, excellent signal level is seen at areas close to the BS. It is also observed that some areas though close to BS experience shadowing leading to low signal level. This can be attributed to the building in the neighborhood of the BS.

The cumulative density function (CDF) of the RSSI is represented in Figure 3 for three sites. The RSSI values are obtained over a large range of distance. We observed in Figure 3 that the two sites (A and B) that are located in the same geographical area and thus having the same terrain tend to behave similarly in statistical sense than the other site (Site C). This difference in behavior can be explained using the climatic or slight different in weather conditions. The city where site $\mathrm{C}$ is located is largely a coastal region compare to that of sites A and B. Although it hardly rains in both cities, City $\mathrm{C}$ is mostly humid. From this figure, it can be observed that there will be less accurate link budget calculation if the same path loss model is adopted for the two different cities without appropriately adapting the model to the environments. For instance, it will be found that for RSSI of about $-70 \mathrm{dBm}$, the city $\mathrm{C}$ link budget will be about $10 \%$ of the time inaccurate (if the same model is used without adapting it), compared to that of other sites $\mathrm{A}$ and $\mathrm{B}$.

Table 1. WiMAX parameters and system settings.

\begin{tabular}{ll}
\hline \multicolumn{1}{c}{ Parameter } & \multicolumn{1}{c}{ Value } \\
\hline Air interface (Multiple access) & OFDM(OFDMA) \\
BS transmit power $(\mathrm{dBm})$ & 23 \\
Carrier frequency $(\mathrm{GHz})$ & 2.5 \\
Total bandwidth $(\mathrm{MHz})$ & 5 \\
No of subcarrier & 512 \\
Duplexing & FDD \\
\hline
\end{tabular}

${ }^{1}$ Note that the results presented in Table 2 are extracted to help illustrate the observed network behavior.
Table 2 shows some recorded snapshots of the network statistics (averaged over a number of samples) for one site ${ }^{1}$. The significance of this table is to demonstrate the impact of interference dynamics when analyzing the performance of the network. It is observed that the rates are quite good for distance with $700 \mathrm{~m}$ from the BS. One thing that should be noted from these results is that the received signal strength indicator (RSSI) alone is not enough to represent the network performance as previously observed in [7]. It is observed that a rate of 7.270 Mbps is obtained for RSSI equal to $-76.5 \mathrm{dBm}$ whereas for a seemingly better RSSI (of $-72 \mathrm{dBm}$ ), a lower rate of $6.080 \mathrm{Mbps}$ is recorded. The explanation is that RSSI does not directly take the interference situation into account. Therefore, RSSI may be very good but the interference could also be very high to produce a low effective signal to-noise plus interference ratio (SINR). It is known that SINR ultimately determines the achievable rate. However, the RSSI is an important parameter for deriving the path loss model [7]. We now embark on the analysis and model fitting for the collected data.

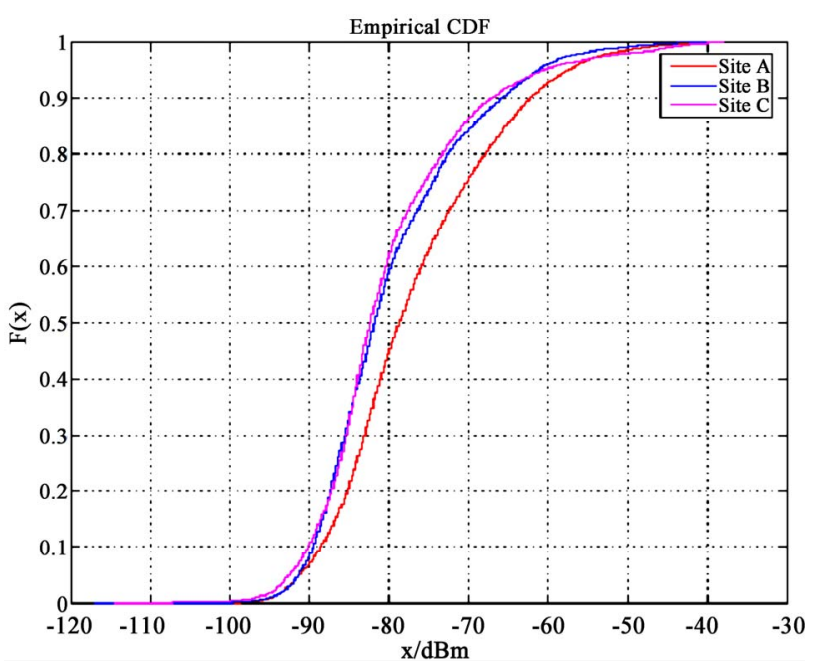

Figure 3. The CDF of the RSSI for the three sites.

Table 2. A site RSSI and measured throughput.

\begin{tabular}{ccc}
\hline Throughput (Mpbs) & RSSI (dBm) & Distance $(\mathrm{m})$ \\
\hline 7.21 & -67.5 & 710 \\
7.27 & -76.5 & 678 \\
6.08 & -72.0 & 675 \\
6.76 & -64.0 & 674 \\
6.09 & -69.5 & 624 \\
3.19 & -77.5 & 503 \\
6.98 & -63.5 & 436 \\
6.13 & -69.0 & 432 \\
7.21 & -65.5 & 413 \\
\hline
\end{tabular}




\subsection{Scattered Plots, Curve Fitting and Results Analysis}

Figure 4 through Figure 6 show the scattered plots of the measured path loss for a number of cell sites, Site 1, Site 2 and Site 3. The free space path loss (FSPL) and the Walfisch-Ikegami propagation model are also shown in the figures. We denote Walfisch-Ikegami model as WIM. The curve fitting (to the measured data) was done in the least square sense (in the log-log scale) using the MATLAB software. We observed that WIM could be used as an approximate model in the absence of our newly derived model.

The variation of the path-loss introduced by the shadowing effects is seen in these scatter plots. The path loss is obtained as, $\mathrm{PL}=\mathrm{Pt}+\mathrm{Gt}+\mathrm{Gr}-\mathrm{RSSI}-$ Losses,

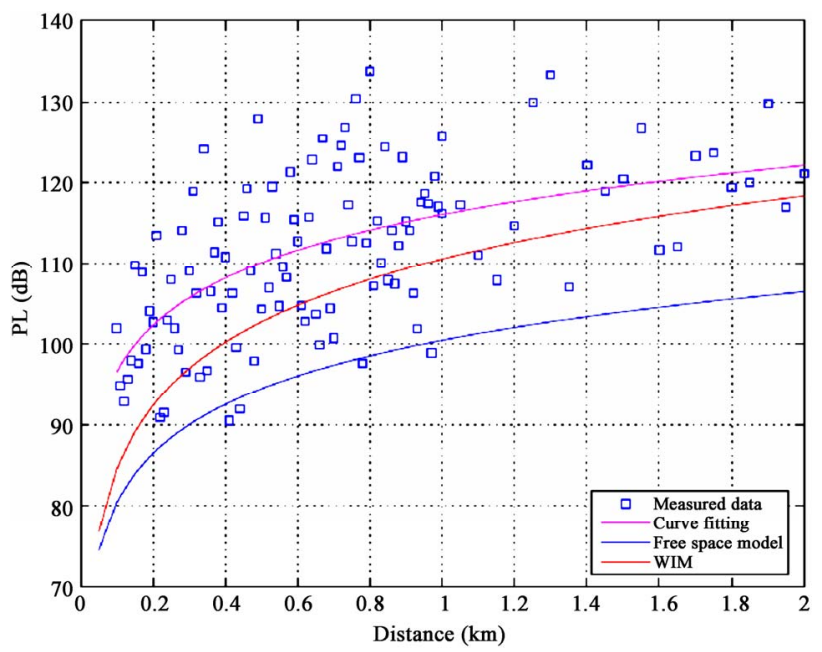

Figure 4. The scatter plots of path loss and curve fitting for site 1.

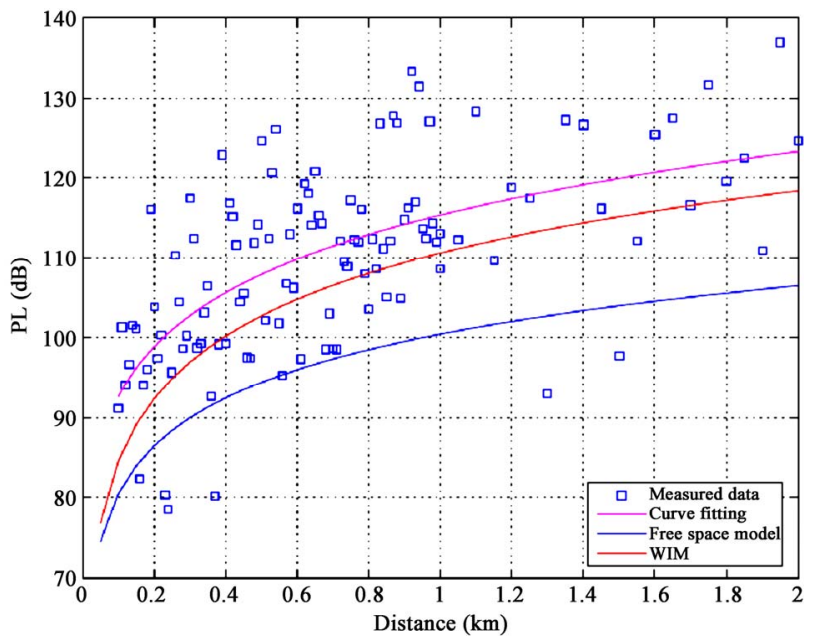

Figure 5. The scatter plots of path loss and curve fitting for site 2. where Gt, Gr, and Losses are respectively, the base station gain $(10 \mathrm{dBi})$, the receive terminal gain $(0 \mathrm{~dB})$, and connection losses $(3 \mathrm{~dB})$. Performing curve fitting to derive path loss model will be less accurate if the signal variation is not smoothen out. To towards that end, we conduct measurements by taking a number of readings at a particular location and obtained the average. We refer to this approach as filtering (i.e., filtering the variations). This implies that we take the average path loss PL = $\mathrm{E}[\mathrm{PL}]$, where $\mathrm{E}[\cdot]$ denotes averaging operators. Curve fitting is performed on the obtained (measured) data and the values for the path loss exponent $(n)$ derived from the fitting are tabulated in Table 3. Both the filtered and unfiltered model parameters are shown.

Figure 7 shows the model fitting for unfiltered data from three sites using log-log scale. It is seen that a significant variation exists among the three different measured data.

Thus, it is difficult to accept any of the models as a good representative of the environment. Figure 8 however shows that by filtering the data, a significant similarity is observed among the data. The fittings for the three sites, except for a negligible difference, are more or

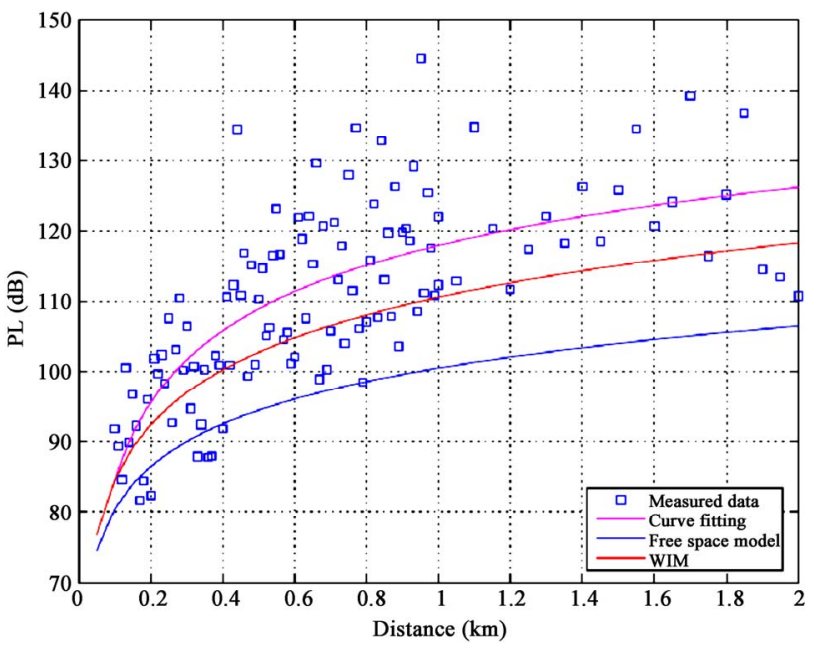

Figure 6. The scatter plots of path loss and curve fitting for site 3.

Table 3. The empirical path loss exponent $n$.

\begin{tabular}{ccccc}
\hline & \multicolumn{2}{c}{ Path loss } & \multicolumn{2}{c}{ Intercept } \\
\hline & $\begin{array}{c}\text { Unfiltered } \\
\text { data }\end{array}$ & $\begin{array}{c}\text { Filtered } \\
\text { data }\end{array}$ & $\begin{array}{c}\text { Unfiltered } \\
\text { data }\end{array}$ & $\begin{array}{c}\text { Filtered } \\
\text { data }\end{array}$ \\
\hline Site 1 & 3.1702 & 2.8259 & 117.9715 & 115.0860 \\
Site 2 & 2.3108 & 2.5117 & 115.3560 & 115.2515 \\
Site 3 & 1.9737 & 2.6993 & 116.0743 & 115.3619 \\
Average & 2.4849 & 2.6790 & 116.4673 & 115.2331 \\
\hline
\end{tabular}




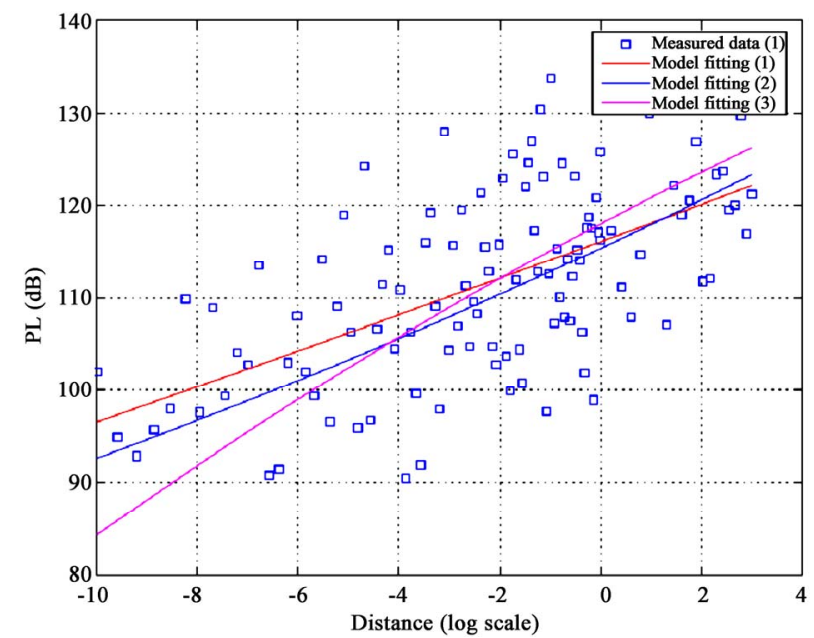

Figure 7. The unfiltered data scatter plot and curve fitting for the path loss.

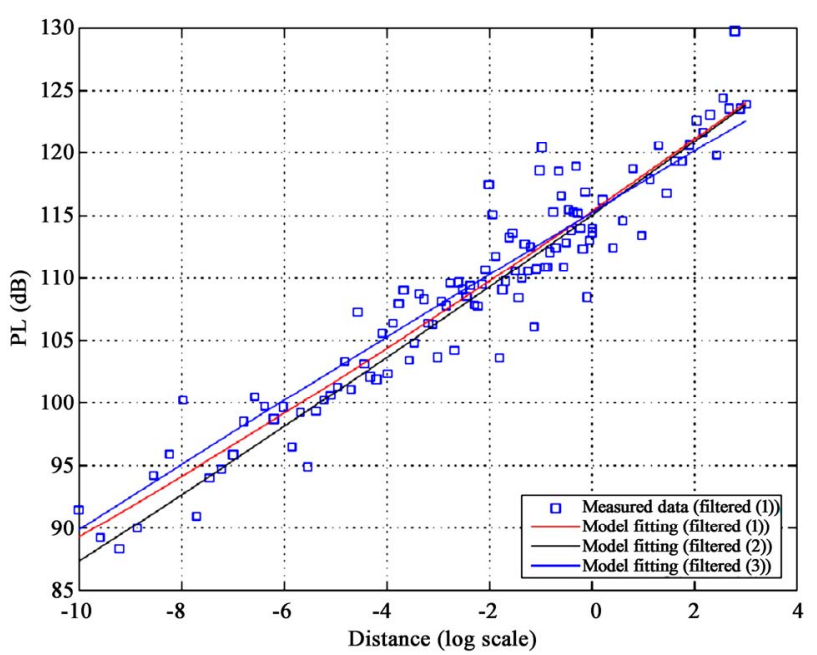

Figure 8. The filtered data scatter plot and curve fitting for path loss.

less the same. Note that in both Figure 7 and Figure 8 the scatter points are shown for only one site in order not to congest the figures. The other findings are presented in Table 3.

It is observed from Table 3 that the intercepts from both the filtered and unfiltered data are very close to each other. However, the intercepts from the filtered data are almost the same. As for the path loss exponent, the filtered data produces (as expected) a representative value. Thus, the average intercept and the average path loss exponent (of the filtered data) are chosen to model the propagation characteristics of the terrain. Hence, the following expression is obtained:

$$
\operatorname{PL}(d)=115.23+26.79 \log _{10}(d)
$$

and it represents a good model for the terrains. The ac- curacy of this model is investigated in the next section through goodness fit.

\subsection{Goodness Fit}

To investigate the goodness fit for the model to the measured data and to also compare the new model with the common/close models, we embark on the following statistical tests. We consider two criteria for examining the goodness fit; the root mean square error (RMSE) and the coefficient of determination $\left(\mathrm{R}^{2}\right)$. The following expression is used to calculate the RMSE

$$
\mathrm{RMSE}=\sqrt{\frac{1}{M} \sum_{i=1}^{M}\left(y_{i}-\hat{y}_{i}\right)^{2}}
$$

where $\hat{y}_{i}$ denotes the estimate of data $y_{i}$ and $M$ is the data length. The statistical measure $\mathrm{R}^{2}$ on the other hand is given as

$$
\mathrm{R}^{2}=1-\frac{\sum_{i=1}^{M}\left(y_{i}-\hat{y}_{i}\right)^{2}}{\sum_{i=1}^{M}\left(y_{i}-\bar{y}_{i}\right)^{2}}
$$

where $\bar{y}_{i}$ is the mean of the measured data. The results in Tables 4-5 show the RMSE for the filtered and unfiltered data, respectively. Given the lower RMSE recorded, it is concluded that the new model shows a better fit to the filtered data compared to other models.

For the unfiltered data the models show poor match, although the new model still shows a better fit.

The calculated values for $\mathrm{R}^{2}$ are shown in Table $\mathbf{6}$ for the new model. Simply put, the coefficient of determination $\left(\mathrm{R}^{2}\right)$ represents the percentage of the data that is closest to the fitted curve. For instance, $\mathrm{R}^{2}=0.9031$ (for the new model for Site 2), implies that about $90 \%$ of the total variation in the measured data can be explained by

Table 4. The average root mean square error for filtered data.

\begin{tabular}{lccc}
\hline & New Model & WIM & $\begin{array}{c}\text { Free Space } \\
\text { Model }\end{array}$ \\
\hline Site 1 & 2.8587 & 5.6399 & 13.9703 \\
Site 2 & 2.7004 & 5.3174 & 13.7131 \\
Site 3 & 2.7952 & 4.8595 & 13.2062 \\
\hline
\end{tabular}

Table 5. The average root mean square error for unfiltered data.

\begin{tabular}{cccc}
\hline & New Model & WIM & $\begin{array}{c}\text { Free Space } \\
\text { Model }\end{array}$ \\
\hline Site 1 & 9.3470 & 10.6977 & 16.8344 \\
Site 2 & 8.8637 & 10.9561 & 17.7060 \\
Site 3 & 9.5029 & 11.2039 & 17.8007 \\
\hline
\end{tabular}


Table 6. The coefficient of determination for the new model.

\begin{tabular}{ccc}
\hline & Filtered Data & Unfiltered Data \\
\hline Site 1 & 0.8773 & 0.3709 \\
Site 2 & 0.9031 & 0.2452 \\
Site 3 & 0.9046 & 0.4960 \\
\hline
\end{tabular}

the linear relationship between the estimated and measured data (as described by the regression equation). The remaining about $10 \%$ of the total variation in the measured data is unexplained. Thus, the coefficient of determination is a measure of how well the fitted curve represents the data. Considering the results in Table 5 the fitted model represents excellent fit to the measured data given that over $87 \%$ of the measured data are explained using the new model. Note that the coefficient of determination is not shown for WIM and FSPL since they are not derived from the data (and not obtained through least square fit).

We make a final remark on the path loss exponent that is obtained for the desert terrain. From the collected data and fitting, we found that $n$ is approximately 2.7. This value is worse than the free space value $(n=2)$ but better than 3 and 3.5 that is usually assumed for urban and shadowed urban, respectively [13]. This is a significant finding or observation. The plain nature and clear sky of the desert environment could be the reason why the path loss exponent is less than that of typical urban or shadowed urban terrains.

\subsection{The Standard Deviation of Shadowing Phenomenon}

Here we confirm through field measurements that shadowing (the relatively long-term fading) is truly log-normal. The histogram shown in Figure 9 exhibits the bell-shaped of a random variable is that normally distributed. The parameter (i.e., standard deviation) of this fading phenomenon is evaluated for the terrain under study. The following expression, modified from [13] is employed for evaluating this parameter.

$$
\sigma=\left|\frac{\overline{\mathrm{PL}}-\gamma}{\sqrt{2} \operatorname{erfcinv}(2 \operatorname{CDF}(\gamma))}\right|
$$

where $\overline{\mathrm{PL}}$ is the average path loss, $\gamma$ and $\operatorname{CDF}(\gamma)$ are arbitrary path loss and the density function of $\gamma$.

From the CDF of the path-loss values (the type shown in Figure 11) the standard deviation can be calculated through appropriate mapping. The data for this part of the study are captured at different locations designated as $d=\{450 \mathrm{~m}, 600 \mathrm{~m}, 900 \mathrm{~m}, 1200 \mathrm{~m}\}$. Figure 10 shows the distribution of the measured signal RSSI while Figure 11 is a representative CDF of the path loss for calculating the values of the spread (or variance or standard deviation) of the data. The calculated standard deviation values are tabulated in Table 7 .

It is observed that the standard deviation is between 8 and 9. The lowest and highest recorded values are respectively, 8.36 and 8.81 (See Table 7). Therefore, for network design, the knowledge of this fading parameter would aid system designers in their link budget calculation. It provides the necessary guidance for determining the fading margin allowances that will ensure minimal network outage conditions.

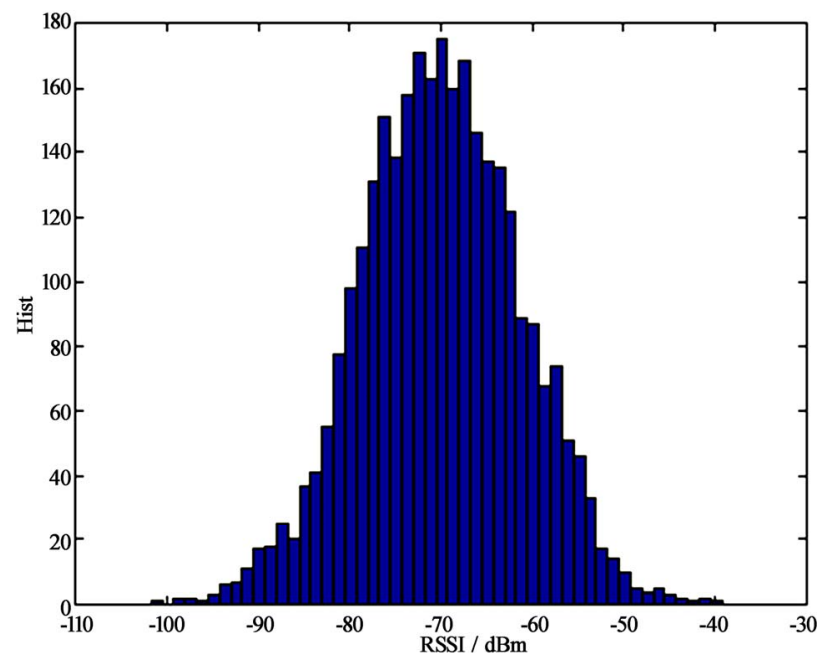

Figure 9. The histogram of the received signal strength indicator at a fixed location, demonstrating the log-normal nature of the shadowing phenomenon.

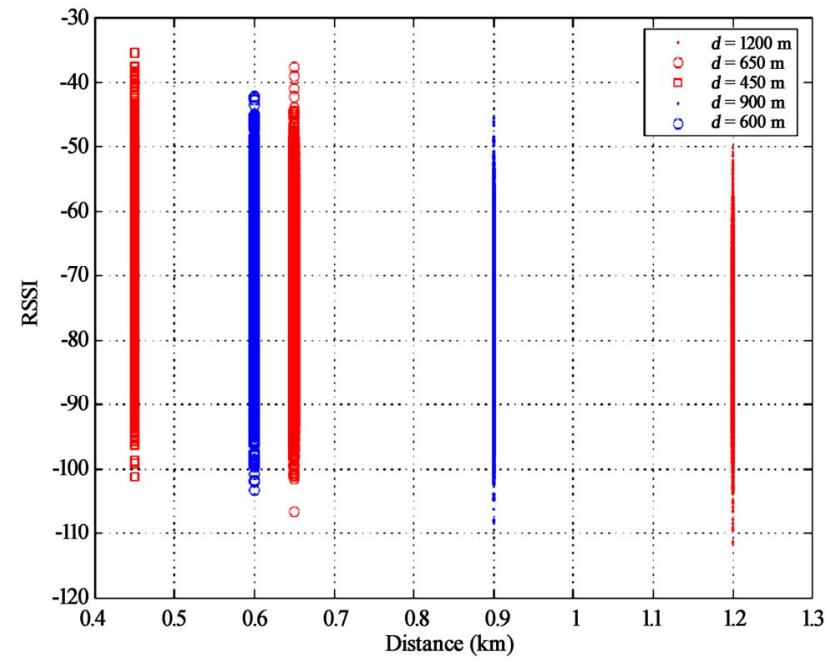

Figure 10. The received signal strength indicator at some fixed locations. The signal level can be mapped to histogram such as the one shown in Figure 9. 
Table 7. Standard deviations of signal variation due shadowing.

\begin{tabular}{cccc}
\hline & \multicolumn{3}{c}{ Standard Deviation } \\
\cline { 2 - 4 } Distance & $d=450 \mathrm{~m}$ & $d=600 \mathrm{~m}$ & $d=900 \mathrm{~m}$ \\
\hline Site 1 & 8.493 & 8.4536 & 8.8114 \\
Site 2 & $\begin{array}{c}8.5944 \\
(d=650 \mathrm{~m})\end{array}$ & 8.7441 & $\begin{array}{c}8.3613 \\
(d=1200 \mathrm{~m})\end{array}$ \\
\hline
\end{tabular}

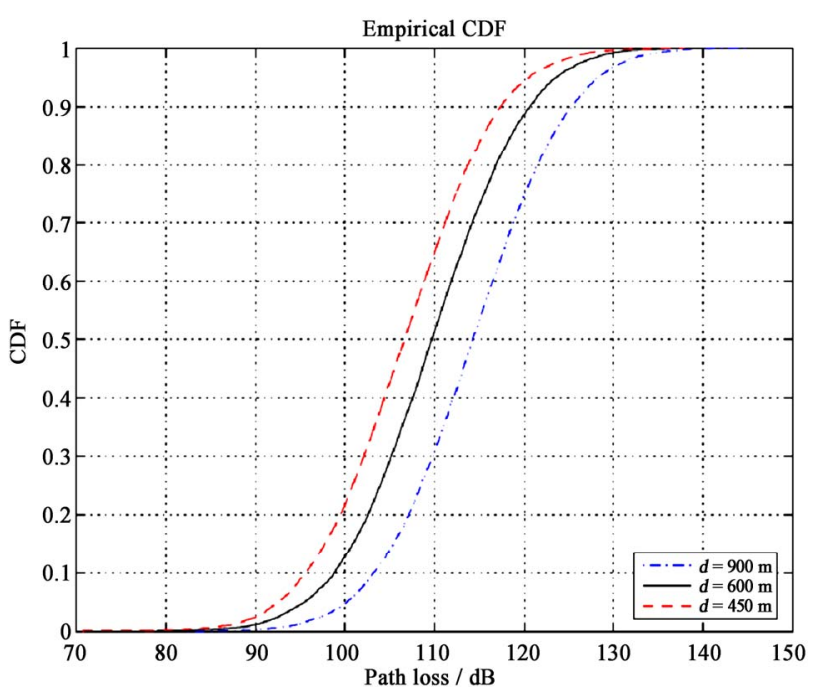

Figure 11. The CDF of the path loss at some fixed locations. This CDF is used in conjunction with (4) to calculate the standard deviation of the shadowing phenomenon.

\section{Conclusion}

This paper presents drive test results for mobile WiMAX network in deserts, cosmopolitan, and fast growing terrains that have not witnessed such measurement efforts. Extensive performance data are collected and analyzed. The propagation behavior is also examined. The path-loss exponent for the desert terrain is obtained which is worse than that of free space but better than that of urban shadowed environment. This is a significant new observation for a terrain that has not witnessed much of this type of study. Thus, our efforts represent a significant contribution to the body of literature in measurement and propagation model in the context of mobile WiMAX systems.

\section{References}

[1] WiMAX. http://www.wimaxforum.org

[2] P. Gronsund, T. Johnsen, P. Engelstad and T. Skeie, "The Physical Performance and Path Loss in a Fixed WiMAX Deployment," Proceedings of the 2007 International Conference on Wireless Communications and Mobile Computing, August 2007, pp. 439-444.

[3] D. De Luca, F. Fiano, F. Mazzenga, C. Monti, S. Ridolfi and F. Vallone, "Outdoor Path Loss Models for IEEE 802.16 in Suburban and Campus-Like Environments," IEEE International Communications Conference, June 2007, pp. 4902-4906.

[4] K. Dongmyoung, C. Hua, N. Minsoo and C. Sunghyun "Performance Measurement over Mobile WiMAX/IEEE 802.16e Network," International Symposium on a World of Wireless, Mobile and Multimedia Networks, June 2008, pp. 1-8.

[5] B. Belloul, A. Aragon-Zavala and S. R. Saunders, "Measurements and Comparison of WiMAX Radio Coverage at 2.5 Ghz and 3.5 Ghz," European Conference on Antennas and Propagation, March 2009, pp. 3287-3291.

[6] E. Nazar, M. A. Salam, A. Al-Lawati, O. Al-Qasmi, M. Al-Gheithi and Z. Nadir, "Modification of an Open Area Okumura-Hata Propagation Model Suitable for Oman," IEEE TENCON, November 2005, pp. 1-4.

[7] J. De Bruyne, W. Joseph, L. Verloock and L. Martens, "Measurements and Evaluation of the Network Performance of a Fixed Wimax System in a Suburban Environment," IEEE International Symposium on Wireless Communication Systems, October 2008, pp. 98-102.

[8] N. LaSorte, W. Barnes, B. Zigreng and H. Refai, "Performance Evaluation of a Deployed WiMAX System Operating in the $4.9 \mathrm{GHz}$ Public Safety Band," IEEE Consumer Communications and Networking Conference, January 2009, pp. 1-5.

[9] V. Teterin, S. Hurley and S. M. Allen, "A Staged Optimization Framework for Cost Optimized Wimax Network Design," The International Conference on Wireless and Mobile Communications, 2008, pp. 185-190.

[10] WINNER. http://www.ist-winner.org

[11] 3GPP, "3rd Generation Partnership Project; Technical Specification Group Radio Access Network; Requirements for Further Advancements for Evolved Universal Terrestrial Radio Access (E-UTRA) (LTE-Advanced) (Release 8)," available online, March 2009.

[12] Z. Nemeth and C. Szabo, "Measurements to Assist Access Network Design with Fixed WiMAX in Urban Environment," ACM International Conference Proceeding, 2006.

[13] T. S. Rappaport, "Wireless Communications: Principles \& Practice," Prentice Hall, New Jersey, 2002. 